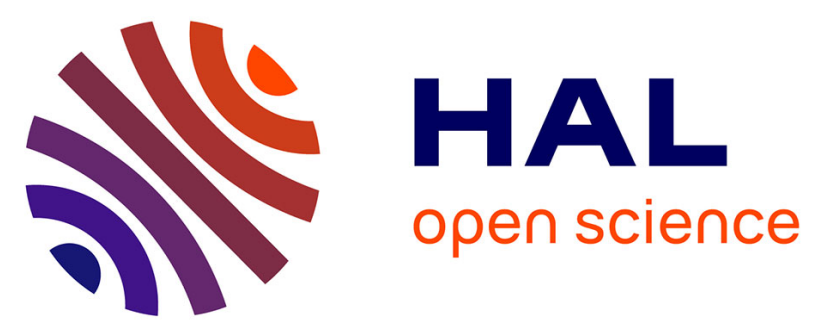

\title{
Détermination de la masse d'eau et des propriétés optiques d'une feuille à partir de modifications de son bilan énergétique. II. - Applications en conditions artificielles et naturelles
}

Galileo Adeli Buriol, Yvette Menoux, Sané de Parcevaux

\section{To cite this version:}

Galileo Adeli Buriol, Yvette Menoux, Sané de Parcevaux. Détermination de la masse d'eau et des propriétés optiques d'une feuille à partir de modifications de son bilan énergétique. II. - Applications en conditions artificielles et naturelles. Agronomie, 1984, 4 (6), pp.501-506. hal-00884665

\section{HAL Id: hal-00884665 https://hal.science/hal-00884665}

Submitted on 1 Jan 1984

HAL is a multi-disciplinary open access archive for the deposit and dissemination of scientific research documents, whether they are published or not. The documents may come from teaching and research institutions in France or abroad, or from public or private research centers.
L'archive ouverte pluridisciplinaire $\mathbf{H A L}$, est destinée au dépôt et à la diffusion de documents scientifiques de niveau recherche, publiés ou non, émanant des établissements d'enseignement et de recherche français ou étrangers, des laboratoires publics ou privés. 


\section{Détermination de la masse d'eau et des propriétés optiques d'une feuille à partir de modifications de son bilan énergétique. II. - Applications en conditions artificielles et naturelles.}

Galileo Adeli BURIOL, Yvette MENOUX $\left(^{*}\right)$ \& Sané de PARCEVAUX (*)

Centro de Ciencias rurais, Universidad federal de Santa Maria, Santa Maria, BR Bresil

(*) I.N.R.A., Station de Bioclimatologie, Route de Saint-Cyr, F 78000 Versailles

RÉSUMÉ Les expériences sont effectuées sur tournesol et sur vigna.
Les résultats montrent que la méthode utilisée, relativement ponctuelle, permet d'étudier de façon fine, précise
et non destructive le comportement de la feuille au cours d'un dessèchement suivi d'une réhumectation. Il est
aisé, entre autres, de suivre l'évolution du contenu en eau respectif du mésophylle et des nervures. L'absorption
foliaire de rayonnement visible $(310$ à $760 \mathrm{~nm})$ est indépendante de la masse d'eau jusqu'à un déficit de
$40-45$ p. 100 mais, dans l'infrarouge $(>760 \mathrm{~nm})$, elle dépend fortement de la masse d'eau foliaire.

Mots clés additionnels : Cinétique thermique, limbe foliaire, rayonnements visible et infrarouge, tournesol, vigna.

Determination of the water content and optical properties of a leaf from changes in its energy balance. II. - Applications under natural and artificial conditions.

Experiments were conducted on sunflower and cowpea plants. The non destructive method used allowed leaf behaviour to be studied accurately during water stress and recovery : in particular, it was easy to follow change in water content separately in the mesophyll and nerves. Leaf absorption in the visible range (310 to $760 \mathrm{~nm}$ ) was also found to be independent of leaf water content up to a water deficit of $40-50 \%$. However, in the infrared range, leaf absorption heavily depended on leaf water content.

Additional key words : Thermokinetics, leaf lamina, visible and infrared radiation, sunflower, cowpea.

\section{INTRODUCTION}

La méthode des cinétiques thermiques ouvre, grâce à sa "non-destructivité ", un certain nombre de possibilités d'études dans le domaine de la physiologie de l'eau des feuilles en conditions artificielles et naturelles (PARCEVAUX, 1972 $a, 1972 b$; SANTIBAÑEZ, 1974 ; BURIOL et al., 1984). Elle permet aussi d'étudier certaines propriétés optiques foliaires et notamment de déterminer de façon directe l'énergie absorbée dans le visible.

L'illustration de l'intérêt de cette méthode dans ce domaine important pour une meilleure estimation de la productivité végétale porte, d'une part, sur la déter- mination de l'hétérogénéité spatiale de la masse d'eau d'une feuille plus ou moins déshydratée, sur l'évolution temporelle du déficit hydrique des feuilles en cours de dessèchement et de réhumectation et, d'autre part, sur la mesure en fonction de l'état hydrique de la feuille de l'absorption des flux de rayonnements visible et infrarouge.

\section{MATÉRIEL ET MÉTHODES}

Les expériences sont effectuées essentiellement sur des feuilles de tournesol (Helianthus annuus L., hybride 6501 I.N.R.A.) et de vigna (Vigna unguiculata (L.) Walpers). 
Au laboratoire, les plantes sont cultivées dans des pots en plastique opaque, sur substrat sableux (PREVOT, 1974 ; BOYER, 1975) avec apport de solution nutritive (COÏC \& LESAINT, 1975). Les conditions climatiques sont les suivantes: rayonnement global moyen au niveau supérieur des plantes de $210 \mathrm{~W} \cdot \mathrm{m}^{-2}$, photopériode de $15 \mathrm{~h}$, température de l'air de $20^{\circ} \mathrm{C}$ $\left( \pm 0,5^{\circ} \mathrm{C}\right)$ le jour, $18{ }^{\circ} \mathrm{C}\left( \pm 0,5^{\circ} \mathrm{C}\right)$ la nuit, humidité relative de l'air respectivement de $66( \pm 2)$ p. 100 et 93 $( \pm 2)$ p. 100. Une période de sécheresse est imposée à un certain nombre de plantes $27 \mathrm{j}$ après la levée. Ces plantes ne reçoivent qu'une quantité d'eau égale à $1 / 2$ ou 1/4 de l'ETM déterminée chaque jour par gravimétrie à partir des plantes constamment irriguées. Après $10 \mathrm{j}$ de sécheresse, les pertes en eau des plantes traitées sont intégralement compensées.

\section{A. Détermination de la masse d'eau surfacique foliaire}

Pour tester l'hétérogénéité spatiale du contenu en eau dans une feuille, on procède à une détermination simultanée par gravimétrie et par la méthode des cinétiques thermiques. En fin de mesure par la méthode des cinétiques thermiques et à chaque endroit de mesure, un disque de $2,5 \mathrm{~cm}^{2}$ est prélevé à l'aide d'un emporte-pièce. Le disque est pesé immédiatement après prélèvement puis après séchage à $100^{\circ} \mathrm{C}$. On en déduit la masse d'eau surfacique Me.

\section{B. Détermination de l'absorption foliaire du rayon- nement}

L'utilisation de filtres appropriés interposés dans le système d'éclairage entre la lampe et la feuille permet l'obtention de lumière de différentes longueurs d'onde: visible $(310$ à $760 \mathrm{~nm})$ et infrarouge (> $760 \mathrm{~nm}$ ).

La méthode utilisée autorise des mesures absolues du flux absorbé par la feuille. Rappelons que la détermination de la pente à l'origine de la cinétique thermique foliaire $(\Delta T / \Delta t)$, de la masse d'eau $M e$ et de la matière sèche surfacique permet alors le calcul de la densité de flux absorbé $\Delta \phi \mathrm{a}$, selon les équations :

$$
\begin{aligned}
\mathrm{MC} & =\mathrm{Me} \cdot \mathrm{Ce}+\mathrm{Ms} \cdot \mathrm{Cs}, \\
\Delta \phi \mathrm{a} & =\mathrm{MC}(\Delta \mathrm{T} / \Delta \mathrm{t}), \\
\text { avec } \mathrm{Cs} & =1250 \mathrm{~J} \cdot \mathrm{kg}^{-1} \cdot{ }^{\circ} \mathrm{C}^{-1} \mathrm{et} \\
\mathrm{Ce} & =4180 \mathrm{~J} \cdot \mathrm{kg}^{-1} \cdot{ }^{\circ} \mathrm{C}^{-1} .
\end{aligned}
$$

Un papier buvard de $0,3 \mathrm{~mm}$ d'épaisseur et de surface rectangulaire $(4,0 \times 5,8 \mathrm{~cm})$, sec et noirci de façon la plus homogène possible avec de l'encre de chine indélébile, a servi de référence pour suivre l'absorption du rayonnement visible d'une feuille de tournesol en voie de dessèchement (fig. 1).

\section{RÉSULTATS ET DISCUSSION}

\section{A. Etude de la masse d'eau surfacique foliaire}

\section{Hétérogénéité spatiale}

Pour une feuille donnée, le coefficient de variation de la masse d'eau surfacique est de l'ordre de 5 p. 100 de la valeur moyenne. Cette hétérogénéité n'évolue pas de façon significative lors d'un dessèchement:

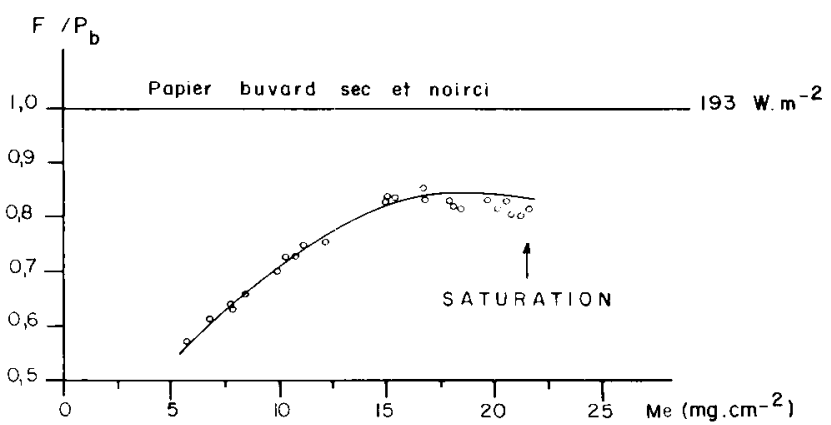

Figure 1

Rapport entre l'absorption du rayonnement visible d'une feuille de tournesol $(F)$ au cours de sa déshydratation et d'un papier buvard sec et noirci avec de l'encre de Chine indélébile $\left(P_{b}\right)$.

Absorption of visible radiation in a dehydrating sunflower leaf $(F)$ as compared to that of a black-ink stained dry blotting paper $\left(P_{b}\right)$.

chez le tournesol, par exemple, la masse d'eau moyenne est de $25,3 \pm 1 \mathrm{mg} . \mathrm{cm}^{-2}$ pour un déficit hydrique de $5 \mathrm{p} .100$ et de $20,0 \pm 0,8 \mathrm{mg} . \mathrm{cm}^{-2}$ pour un déficit hydrique de 21 p. 100.

Cette variabilité n'est pas due au hasard, mais à la variation spatiale normale de la masse d'eau dans la feuille : les valeurs les plus élevées se trouvent près de la base et de la nervure centrale où le limbe est le plus épais chez le tournesol, dans les folioles latérales chez le vigna. Pour une estimation globale du contenu en eau de la feuille, il conviendra d'en tenir compte en choisissant convenablement les points de mesure.

\section{Evolution temporelle de la masse d'eau foliaire}

Comme le montre la figure 2 , il est aisé de suivre l'évolution de l'état hydrique d'une feuille in situ alors que la plante est soumise à une sécheresse édaphique puis irriguée de nouveau : à chaque variation dans l'alimentation en eau des racines, on observe une réponse quasi instantanée au niveau de la feuille.

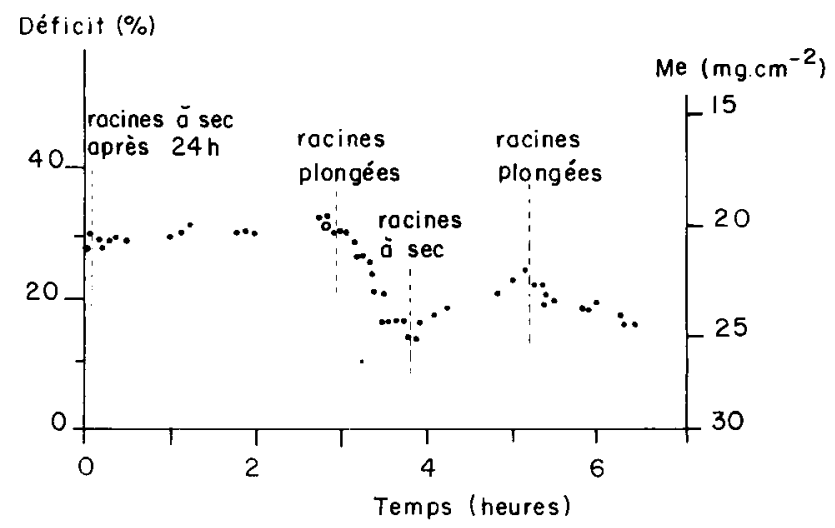

Figure 2

Variation de la masse d'eau d'une feuille de tournesol après modifications de l'alimentation hydrique au niveau des racines. La masse d'eau à saturation est déterminée sur des disques foliaires prélevés à la fin de l'expérience, en utilisant la méthode de ĊATSKÝ (1965), par flottaison sur eau distillée.

Variation in the water content of a sunflower leaf following modifications in the water supply to the roots. The water content at saturation was determined on leaf disks collected at the end of the experiment using the method of $\check{C}_{A T S K Y}(1965)$ by floating on pure water. 
De plus, dans le cas d'une feuille excisée, la détermination simultanée de la masse d'eau du mésophylle par la méthode des cinétiques thermiques et de la masse d'eau totale du limbe par gravimétrie permet de dissocier le comportement hydrique du mésophylle de celui des nervures principales (fig. 3). Contrairement à ce qu'on peut attendre, le mésophylle se déshydrate plus vite que les nervures.

Cette méthode peut donc utilement compléter les études sur les variations du stock d'eau présent dans les différents " compartiments » d'une plante (CRUIZIAT, 1972, 1973, 1974).

\section{B. Etude de l'absorption du flux de rayonnement par les feuilles}

1. Etude comparative de l'absorption foliaire dans le visible et l'infrarouge en fonction de l'état hydrique de la feuille (fig. 4)

Entre la saturation et $40-45$ p. 100 de déficit on n'observe pas de variations de l'absorption de la lumière visible. Au-delà de cette limite, l'absorption décroît en fonction de l'état hydrique du limbe : à 50 p. 100 de déficit, l'absorption est réduite de 10 p. 100.

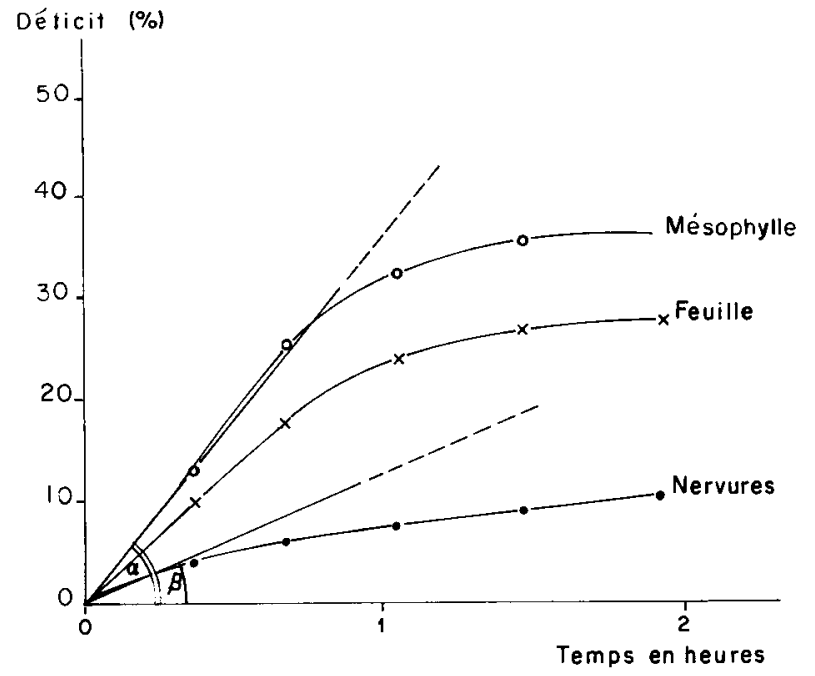

Figure 3

Dessèchement de la feuille entière, des nervures et du mésophylle d'une même feuille de tournesol après son excision et soumise à un fort éclairage (lampe Phillips spot $13011 \mathrm{E}$. $150 \mathrm{~W}$ à $1,5 \mathrm{~m}$ environ de la feuille).

Dehydration of the whole leaf, nerves and mesophyll of a sunflower leaf after excision, under strong light (Spotlight Philips 13011 E. 150 $W$ located about $1.5 \mathrm{~m}$ away from the leaf).

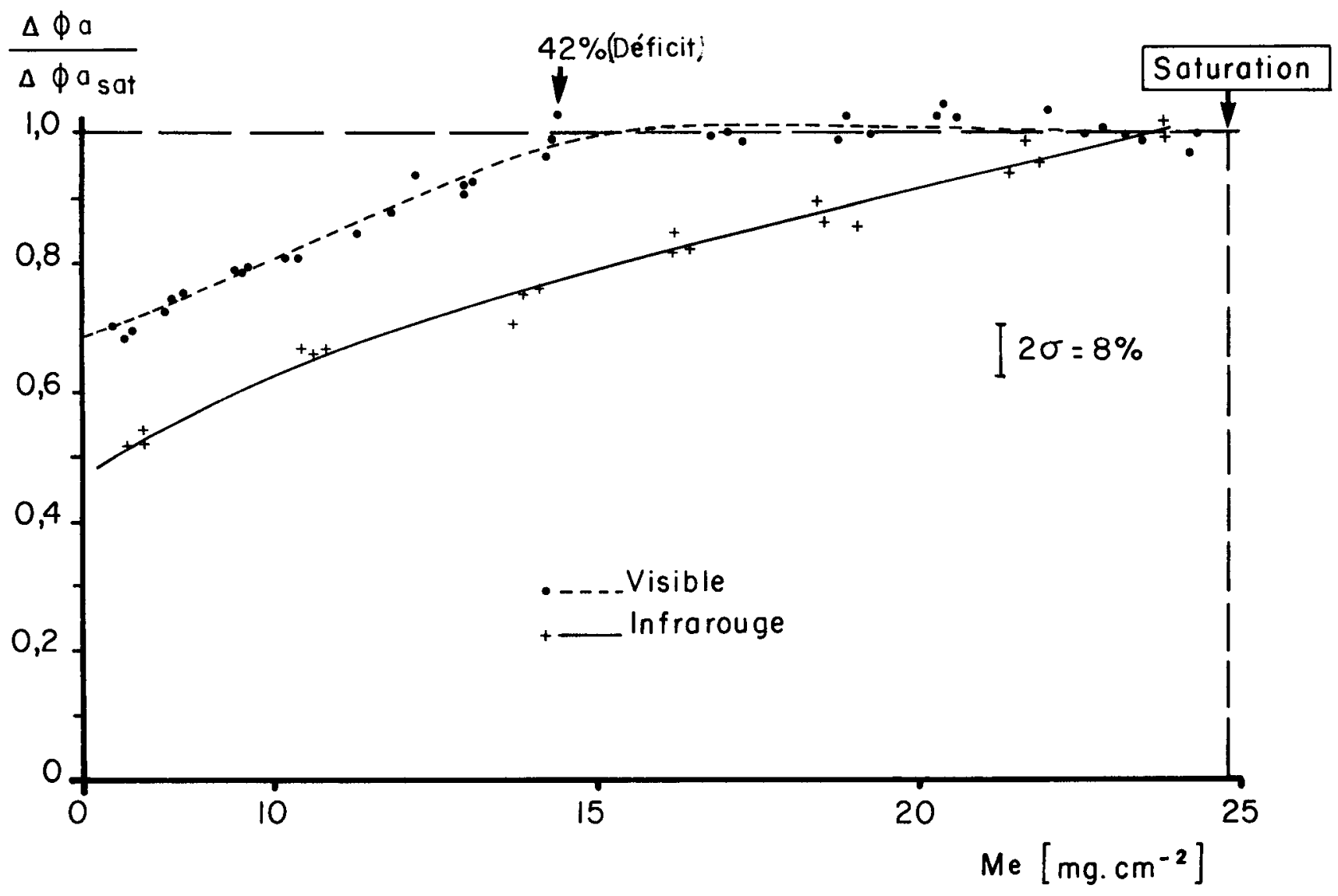

Figure 4

Evolution de l'absorption du flux de rayonnement visible et infrarouge, exprimée en $p .100$ de la valeur à saturation en fonction de la masse d'eau surfacique (Me) d'une feuille de tournesol.
Change in the absorption of the visible and infrared radiation flux as a percentage of the value at saturation, in relation to the surface water content (Me) of a sunflower leaf. 
Ces résultats obtenus pour le tournesol dans le visible sont conformes à ceux trouvés dans la littérature, déduits de la réflexion et de la transmission, pour d'autres espèces (THOMAS et al., 1966 ; GAUSMAN et al., 1971).

Dans l'infrarouge, la densité du flux absorbé dépend fortement de l'état hydrique du limbe. L'absorption diminue d'une façon presque linéaire jusqu'à 40 p. 100 de déficit hydrique, où sa valeur n'est plus que 80 p. 100 de la valeur à pleine turgescence. Au-delà de cette valeur, la variation du flux absorbé n'est plus linéaire et décroît plus rapidement.

Ces résultats sont également conformes à ceux publiés par CARLSON et al. (1971) qui trouvent une bonne corrélation entre la réflexion dans l'infrarouge et le contenu en eau relatif de la feuille.

\section{Absorption foliaire dans le visible}

\section{a) Hétérogénéité spatiale}

Pour une valeur moyenne de $93,0 \mathrm{~W} . \mathrm{m}^{-2}$ obtenue en 10 points d'une feuille de tournesol avec 5 répétitions, l'écart-type est de $3,2 \mathrm{~W} . \mathrm{m}^{-2}$, ce qui représente un coefficient de variation de 3 p. 100. Des résultats semblables se retrouvent pour le vigna. Cette variation, même si elle n'est pas négligeable, est sensiblement inférieure à celle obtenue pour la masse d'eau surfacique. La répartition des valeurs semble aléatoire.

b) Evolution de l'absorption de rayonnement au cours d'une sécheresse et d'une réhumectation du sol (fig. 5)

Il n'y a pas de différences significatives dans cette évolution pour l'ensemble des feuilles de vigna pendant la sécheresse et la réhumectation. Par contre, chez le tournesol, l'absorption est moins élevée pour les feuilles des plantes ayant subi la sécheresse, particulièrement pour les feuilles les plus âgées. Lors de la réhumectation, cette différence subsiste pour les feuilles les plus âgées, alors qu'elle s'efface pour les feuilles les plus jeunes.

Les résultats obtenus pour le tournesol sont en accord avec ceux trouvés pour d'autres espèces (KNIPLING, 1970 ; CARLSON et al., 1971 ; SINCLAIR et al., 1971 ; WOLLEY, 1971 ; GAUSMAN \& AlLEN, 1973 ; GAUSMAN et al., 1973 ; RODE \& BETHENOD, 1980). Les résultats obtenus pour le vigna confirment ceux trouvés par ROY \& METHY (1980) pour des graminées où un déficit hydrique modéré du sol n'entraîne pas de différence significative dans la transmission et la réflexion du rayonnement visible. Ces phénomènes sont probablement liés à l'état hydrique des feuilles : sous l'effet de la sécheresse, les feuilles du tournesol traité ont subi une forte déshydratation. Cette déshydratation ne s'est pas produite dans les feuilles de vigna.

\section{c) Influence $d u$ rang de la feuille sur la tige (tabl. 1 et 2)}

La variation de la densité du flux absorbé n'est pas significative en conditions artificielles, aussi bien pour le tournesol que pour le vigna. Chez le tournesol cultivé en conditions naturelles et à un stade phénologique plus avancé, l'absorption varie fortement entre les

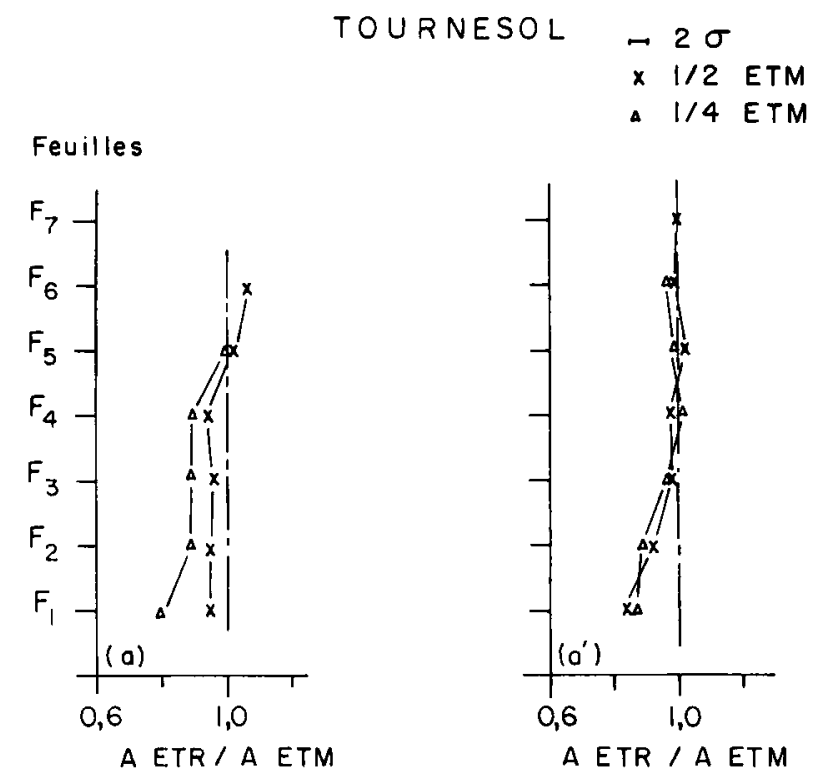

VIGNA

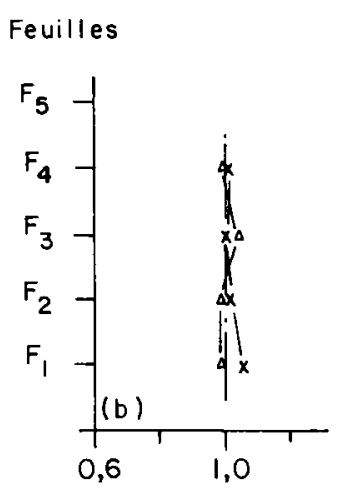

A ETR / AETM

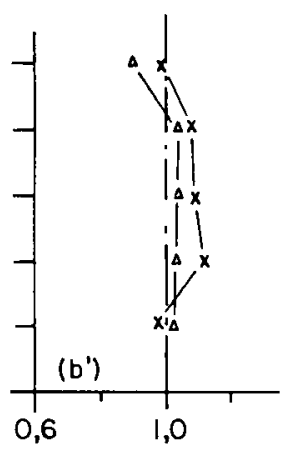

A ETR / AETM
Figure 5

Absorption de la densité $d u$ flux du rayonnement visible pour les feuilles de vigna et de tournesol, après 10 et $9 j$ de sécheresse la et $b)$, respectivement, et après $10 j$ de réhumectation $\left(a^{\prime}\right.$ et $\left.b^{\prime}\right)$. Energie incidente $-193 . W \cdot m^{-2}\left(193 \mathrm{~W} \cdot \mathrm{m}^{-2}=100 \mathrm{p}\right.$. 100). Le rang de la feuille sur la tige est compté à partir de la base.

Absorption of the density of the visible radiation flux for cowpea and sunflower leaves after 10 or 9 days of dehydration respectively $(a$ and $b)$ and after 10 days of rehydration $\left(a^{\prime}\right.$ and $\left.b^{\prime}\right)$. Incident radiation : $193 \mathrm{~W} \cdot \mathrm{m}^{-2}\left(193 \mathrm{~W} \cdot \mathrm{m}^{-2}=100 \%\right)$. The lowest leaf on the stem is noted as F1.

feuilles placées à différents niveaux bien qu'elles présentent sensiblement la même masse calorifique.

L'apparition de différences significatives importantes vers la fin du cycle végétatif a déjà été observée par GATES et al. (1965), WONG \& BLEVIN (1967), MCCREE (1972), GATES (1980) même en l'absence de signes visibles de sénescence.

\section{CONCLUSIONS}

Les expériences sur le bilan hydrique des feuilles, après une modification de leur alimentation en eau, mettent en évidence l'intérêt de la méthode préconisée pour une étude de la dynamique de l'eau dans la plante entière. Une réponse rapide, dans le contenu 


\section{TABLEAU 1}

Absorption $\left(W \cdot m^{-2}\right)$ des feuilles placées à différents niveaux sur la tige d'un vigna et sur la tige d'un tournesol cultivés en conditions artificielles et toujours bien alimentés en eau (face supérieure). Le rayonnement incident était de $200 \mathrm{~W} . \mathrm{m}^{-2}$.

Radiation $\left(W . \mathrm{m}^{-2}\right)$ absorbed by leaves at different levels of the stem of flowering sunflower plant grown in artificial conditions and adequately supplied with water (upper surface). Incident radiation $=200 \mathrm{~W} \cdot \mathrm{m}^{-2}$.

\begin{tabular}{lrrrrrr}
\hline \multicolumn{1}{c}{ Feuilles } & $\mathrm{F}_{1}$ & $\mathrm{~F}_{2}$ & $\mathrm{~F}_{3}$ & $\mathrm{~F}_{4}$ & $\mathrm{~F}_{5}$ & $\mathrm{~F}_{6}$ \\
\hline Vigna & & & & & & \\
$\quad$ moyenne & 130,0 & 130,4 & 131,7 & 133,4 & 133,4 & 133,1 \\
$\quad$ écart-type & 4,3 & 2,3 & 3,6 & 4,6 & 2,5 & 3,9 \\
$\begin{array}{l}\text { Tournesol } \\
\text { moyenne }\end{array}$ & 122,2 & 125,6 & 126,0 & 128,3 & 131,1 & 125,1 \\
écart-type & 4,0 & 0,9 & 4,7 & 3,3 & 2,9 & 4,4 \\
\hline
\end{tabular}

\section{TABLEAU 2}

Absorption $\left(W . m^{-2}\right)$ des feuilles placées à différents niveaux sur la tige de tournesol en pleine floraison et cultivé en conditions naturelles (face supérieure). Rayonnement incident $=240 \mathrm{~W} \cdot \mathrm{m}^{-2}$. Radiation $\left(W . \mathrm{m}^{-2}\right)$ absorbed by leaves at different levels of the stem of flowering sunflower plants grown in natural conditions (upper surface). Incident radiation $=240 \mathrm{~W} \cdot \mathrm{m}^{-2}$.

\begin{tabular}{lrrrrrrrr}
\hline \multicolumn{1}{c}{ Feuilles } & $\mathrm{F}_{1}$ & $\mathrm{~F}_{2}$ & $\mathrm{~F}_{3}$ & $\mathrm{~F}_{4}$ & $\mathrm{~F}_{5}$ & $\mathrm{~F}_{6}$ & $\mathrm{~F}_{7}$ & $\mathrm{~F}_{8}$ \\
\hline Absorption & 143,0 & 169,0 & 172,8 & 175,1 & 171,0 & 179,8 & 206,6 & 208,5 \\
Ecart-type & 5,2 & 4,8 & 2,4 & 7,4 & 7,3 & 8,2 & 7,2 & 3,0 \\
\hline
\end{tabular}

hydrique de la feuille, est facilement décelée lors d'un changement brutal dans l'alimentation des racines. De façon analogue, une variation presque instantanée du contenu en eau du mésophylle peut s'observer lorsque la feuille est détachée de la plante.

Quelques aspects nouveaux sur la dynamique de l'eau contenue dans le mésophylle et dans les nervures sont présentés. Le rapport des vitesses de déshydratation mésophylle/nervure peut atteindre des valeurs voisines de 2 quand la feuille se dessèche d'une façon rapide, l'état hydrique du mésophylle évoluant plus rapidement que celui de la feuille entière.

De plus, les feuilles des espèces expérimentées, bien que présentant une certaine hétérogénéité hydrique, se dessèchent d'une façon assez homogène, ceci s'expliquant probablement par l'extension et l'efficience de leur réseau vasculaire. $\mathrm{Ce}$ fait permet de définir une modalité opératoire dans l'application de la méthode : on obtient une approche de l'évolution de l'état hydrique de la feuille entière à partir d'un point bien choisi. Une détermination de la masse d'eau du limbe par prélèvement de disques à la fin de l'expérience permet d'évaluer, en valeur absolue, l'évolution de la masse d'eau du limbe foliaire au cours d'une période.

La méthode des cinétiques thermiques permet une mesure directe du flux de rayonnement absorbé, contrairement aux méthodes existant jusqu'à présent selon lesquelles on déduit le rayonnement absorbé par différence entre rayonnement incident et rayonnement réfléchi et transmis. Elle confirme que la densité du flux absorbé dans le visible, dans les conditions réalisées et pour le matériel végétal utilisé, est indépendante de l'état hydrique entre la saturation et 40-45 p. 100 de déficit. Par contre, l'absorption dans l'infrarouge $(>760 \mathrm{~nm})$ dépend fortement de l'état hydrique de la feuille dans toute la gamme des déficits.

Cette nouvelle technique ouvre donc la voie à de nombreuses investigations tant dans le domaine fondamental qu'appliqué. Au niveau pratique, elle devrait permettre de mieux définir les différentes stratégies mises en place par les végétaux pour s'adapter aux contraintes du milieu, dans la mesure où l'on peut déceler très rapidement l'influence de différentes modalités d'arrosage, de l'intensité de la sécheresse. Elle permet aussi de classer et de sélectionner des espèces ou des variétés plus ou moins résistantes à la sécheresse.

Reçu le 2 février 1983. Accepté le 7 février 1984.

\section{RÉFÉRENCES BIBLIOGRAPHIQUES}

Boyer Y., 1975. Premières études sur les mécanismes de l'accélération de croissance induite par une sécheresse temporaire. $C$. $R$. Acad. Sci., Paris, série D, 280, 283-286.

Buriol G. A., Santibañez F., Menoux Y., Parcevaux S. de, Bertolini J. M., 1984. Détermination de la masse d'eau et des propriétés optiques d'une feuille à partir de modifications de son bilan énergétique. I. - Bases théoriques de la méthode et technique de mesure. Agronomie, 4 (6), 493-500.

Carlson R. E., Yarger D. N., Shaw R. H., 1971. Factors affecting the spectral properties of leaves with special emphasis of leaf water status. Agron. J., 63, 486-489.

Čatský J., 1965. Leaf-disk method for determining water saturation deficit, p. 353-360. In F. E. Eckardt : « Methodology of plant eco-physiology ». UNESCO, Paris.

Coïc Y., Lesaint C., 1975. La nutrition minérale et en eau des plantes en horticulture avancée. Doc. Tech. S.C.P.A., 23, 22 p.

Cruiziat P., 1972. Contribution à l'étude des réserves en eau de la plante. Thèse, Univ. Paris VI, $126 \mathrm{p}$.

Cruiziat P., 1973. Etude du fonctionnement de feuilles en régime de réhumectation. Physiol. Vég., 12, 110-111.
Cruiziat P., 1974. Détermination des pertes en eau subies par les différents organes d'une plante soumise au dessèchement. Ann. Agron., 25, 539-554.

Gates D. M., 1980. Spectral properties of plants, p. 215-248. In D. M. Gates" « Biophysical ecology ». Springer Verlag, 611 p.

Gates D. M., Keegan H. J., Schleter J. C., Weidner V. R., 1965. Spectral properties of plants. Appl. Opt., 4, 11-20.

Gausman H. W., Allen W. A., 1973. Optical parameters of leaves of 30 plant species. Plant Physiol., 52, 57-62.

Gausman H. W., Allen W. A., Escobar D. E., Rodriguez R. R., Cardenas R., 1971. Age effects of cotton leaves on light reflectance, transmittance and absorptance and on water content and thickness. Agron. J., 63, 465-469.

Gausman H. W., Allen W. A., Wiegend C. L., Escobar D. E., Richardson A. L., 1973. The leaf mesophylls of twenty crops, their light spectra and optical and geometrical parameters. U.S.A. Techn. Bull., 1465, 59 p.

Knipling E. B., 1970. Physical and physiological basis for the reflectance of visible and near infrared radiation from vegetation. Remote Sensing Environ., 1, 155-159. 
McCree K. J., 1972. The action spectrum, absorbance and quantum yield of photosynthesis in crop plants. Agric. Meteorol., 9, 191-216.

Parcevaux S. de, 1972a. Contribution à l'étude des échanges gazeux au niveau des feuilles. Thèse Doct. d'Etat, Fac Orsay, 86 p. + annexes.

Parcevaux S. de, 1972 $b$. Aspects biophysiques des échanges entre les feuilles et le milieu environnant. Oecol. Plant., 7, 371-401.

Prevot J. P., 1974. Influence des conditions d'alimentation en eau sur la croissance et le développement du lin. Mémoire fin études Inst. Sup. agric. Beauvais, 56 p.

Rode J. C., Bethenod O., 1980. Influence de la carence hydrique sur le comportement photosynthétique du lin. Ann. Agron., 31, 285-295.

Roy J., Methy M., 1980. Absorption of photosynthetical active radiation by the leaves of grass species : inter and intraspecific variations and phenotypic response to water stress. Acta Oecol., Oecol. Plant., 1 (3), 253-256.

Santibãnez F., 1974. Etude des variations de la teneur en eau des feuilles au moyen d'une méthode rapide et non destructive. Thèse Univ. Paris VII, $78 \mathrm{p}$.

Sinclair T., Hoffer R. M., Schreiber M., 1971. Reflectance and internal structure of leaves from several crops during a growing season. Agron. J., 63, 864-868.

Thomas J. R., Myers V. I., Hielman M. D., Wiegand C. L., 1966. Factors affecting light reflectance of cotton. Symp. Proceed. Inst. Sci. \& Techn. Univ. Michigan, Remote sensing of environment., Vol. 4, 305-312.

Wong C. L., Blevin W. R., 1967. Infrared reflectances of plant leaves. Austr. J. Biol. Sci., 20, 501-508.

Wolley J. T., 1971. Reflectance and transmittance of light by leaves. Plant Physiol., 47, 656-662. 